\title{
The Impact of Preventive Interventions on Intimate Partner Violence among Pregnant Women Resident in Hamadan City Slum Areas Using the PEN-3 Model: Control Randomized Trial Study
}

\author{
Khadije Ezzati Rastegar ${ }^{1}$, Babak Moeini ${ }^{1,2}$, Forouzan Rezapur-Shahkolai ${ }^{1,3}$, Asadollah Naghdi ${ }^{4}$, Manoochehr Karami ${ }^{5}$, \\ Shayesteh Jahanfar,**
}

'Department of Public Health, School of Public Health, Hamadan University of Medical Sciences, Hamadan, Iran ${ }^{2}$ Social Determinants of Health Research Center, Hamadan University of Medical Sciences, Hamadan, Iran ${ }^{3}$ Research Center for Health Sciences, Hamadan University of Medical Sciences, Hamadan, Iran ${ }^{4}$ Department of Social Sciences, Buali Sina University, Hamadan, Iran

${ }^{5}$ Modeling of Non-communicable Diseases Research Center, Hamadan University of Medical Sciences, Hamadan, Iran ${ }^{6}$ Department of Public Health and Community Medicine, Tufts School of Medicine, Boston, MA, USA

Background: Intimate partner violence is a severe life-threatening criminal and public health problem affecting the well-being of individuals, families, and society. Planning interventions to reduce the burden of this persistent and criminal violence should be relevant culturally and socially.

Methods: In this randomized control trial, 150 pregnant women residing in slum areas of Hamadan were randomly assigned to two groups (intervention group: $\mathrm{n}=50$ and control group: $\mathrm{n}=100$ ). Interventional strategies included educating the victims based on local cultural norms, culturally sensitive individual and group counseling, and educating health care providers. Data were collected via face-to-face interviews at baseline and again at 3 months after the intervention. We used a paired t-test to evaluate the effect of the intervention by comparing changes in the outcomes measured.

Results: There were no statistically significant differences between the two groups at baseline in terms of sociodemographic characteristics. Post-test scores of knowledge (7.50 \pm 2.65 vs. $5.14 \pm 3.51, \mathrm{P}=0.001$ ), communication skills (18.38 \pm 4.25 vs. $16.2 \pm 3.83, \mathrm{P}=0.04)$, and family support and social expectation of obedience $(15.79 \pm 4.45$ vs. $13.40 \pm 4.57, \mathrm{P}=0.005$ ) of the victims were statistically significantly higher in the experimental group compared to the control group. Moreover, physical $(0.74 \pm 2.28$ vs. $1.20 \pm 2.60, \mathrm{P}=0.06)$, psychological $(2.80 \pm 4.10$ vs. $4.52 \pm 5.43, \mathrm{P}=0.06)$, and sexual $(0.11 \pm 0.58$ vs. $0.61 \pm 1.22, \mathrm{P}=0.04)$ violence reduced in the experimental group compared to the control group.

Conclusion: Culturally relevant interventions can reduce intimate partner violence.

Keywords: Intimate Partner Violence; Pregnant Women; Slums

Received: May 19, 2020, Revised: June 24, 2020, Accepted: June 25, 2020

*Corresponding Author: Shayesteh Jahanfar https://orcid.org/0000-0001-6149-1067

Tel: +1-617-636-2959, Fax: +1-617-636-4017, E-mail: shayesteh.jahanfar@tufts.edu 


\section{INTRODUCTION}

Intimate partner violence (IPV) is a worldwide public health crisis affecting many women and families. ${ }^{1)}$ Recently, World Health Organization reported that one-third of women globally have experienced physical, sexual, or both types of violence inflicted by their spouse or another sexual partner. Such women undergoing violence show adverse health outcomes such as anxiety, post-traumatic stress disorder, despair, and low self-esteem. Most victims experience health-associated complications, including genital infections, gastrointestinal, neurological, and cardiovascular complications, an increased rate of smoking, and illicit drug use. ${ }^{2,3)}$

The prevalence of IPV during pregnancy differs across the world ranging between $15 \%-71 \%{ }^{4-7)}$ According to an Egyptian study, in $30 \%$ of women, the first experience of IPV occurs during pregnancy, ${ }^{4)}$ causing severe consequences for both the mother and the fetus. ${ }^{3)}$ In a systematic review, an investigation of 32 studies across a number of communities of Iran (15,610 individuals) from 2002 to 2014 indicated that the prevalence of violence, in general, ranged between $43 \%$ and $62 \%$. Physical (19\%), sexual (31\%), and psychological violence (45\%) have also been reported among Iranians. ${ }^{8)}$

Despite all these reports, many women choose to be silent against violence and even try to conceal it. ${ }^{9)}$ For fear of losing their children or being compelled to live away from them and their home if they unveil the harassment, the incidents of insults are concealed and the consequential psychological and physical burden disguised. ${ }^{10)}$

Women's response to IPV stems from their belief systems and lifestyles, which are specifically associated with the culture of a society. ${ }^{11)}$ Pregnant women living in slum areas suffer from poverty, lack of education, and poor health. The fabric of their culture requires a set of values and norms in regions associated with social isolation and seclusion making them even more vulnerable to violence. ${ }^{12)}$ The intervention was developed using a theory-based PEN-3 that is culturally relevant and sensitive to immigrant pregnant women living in Hamadan's slum areas.

The PEN-3 model was developed by professor Airhihenbuwa in 1989 to focus on culture. It consists of three primary domains: health education target groups, relationships and expectations, and cultural empowerment or cultural identity. In fact, the PEN-3 model highlights the impact of behavior on health (positive, existential, or negative), within the broader context of culture to diagnose the roles, values, and norms that either support or do not support various types of activities (perceptions, enablers, or nurturers) and the focus of the health behavior intervention (person, extended family, or neighborhood). ${ }^{13)}$ The current study aims at investigating the impact of preventive interventions based on the PEN-3 model to reduce IPV among pregnant women residing in the slum areas of Hamadan.

\section{METHODS}

\section{Participants}

The present study was part of a larger study aimed at identifying sociodemographic characteristics affecting IPV among pregnant women in suburban Hamadan. ${ }^{14)}$ In Hamadan, which is in the mountainous area of Western Iran, $39 \%$ of the population lives in the suburbs, the majority of whom are immigrants, and $80 \%$ of the female residents are at the reproductive age. This study was conducted in partnership with health care clinics. The data for this interventional study were derived from 150 pregnant women who were referred to three (out of 15 existing) health clinics between January and August 2018. We recruited pregnant women who were 20 years or older, lived with their husbands, were recognized as permanent residents of the slums, and had electronic health records.

\section{Sample Size Determination}

The sample size was determined considering both the experimental and control groups. The differences between the intervention and control groups in a similar study in Iran for reduction of physical violence, before and after intervention respectively, were $18.57 \pm 2.83$ and $21.02 \pm 3.31 .^{15)}$ We considered a $5 \%$ margin of error, a $5 \%$ significance level, and $90 \%$ power. We added $15 \%$ to the computed sample size considering potential attrition $(n=150)$. We recruited subjects using block randomization to reduce bias and ensure a balance between the experimental and control groups. To increase statistical power, the number of samples in the control group was twice that of the experimental group $(n=100)$. The study was explained to the participants prior to the intervention and their written informed consent obtained.

$$
n=\frac{\left(Z_{1-\frac{\alpha}{2}}+Z_{1-\beta}\right)^{2}+\left(\sigma_{1}^{2}+\sigma_{2}^{2}\right)}{\left(\mu_{1}-\mu_{2}\right)^{2}}
$$

\section{Procedures}

Three districts were selected out of 15 slum areas based on the prevalence of IPV, stability of clinics, or mobility of researcher, and feasibility of conducting the intervention. Participants from each clinic were randomly allocated to experimental and control groups. We examined baseline characteristics of the sample; to ensure that key variables (experiences of violence) were indeed evenly distributed in the randomization. This randomization was automated, using computer-generated random numbers.

Participants were blinded to their trial group. The experimental group received the intervention for preventing IPV for 5 weeks, while the control group received none.

Ethical approval was granted by the Ethics Committee in Hamadan University of medical Sciences (approval no., grant IR Umsha. Rec.1396.478). This was an experimental study with a pre-test assessment followed by an educational and consultation intervention and post-test evaluation. We obtained written consent from all the women prior to administering the pre-test phase. We assured the participants 
that all their responses would be kept anonymous and that completing pre- and post-test was voluntary and would not interfere with the type of health services they needed. The women could withdraw from the study at any time during the study.

\section{Instrument}

Data were collected using questions adapted from our prior qualitative study based on the PEN-3 model. ${ }^{14)}$ The domains for this model consist of variables related to knowledge (11 items using a 3-point scale "yes/no/don't know," for example, "does forcing the wife for sex without her consent mean violence?" or "does the betrayal of the husband and his relationship with someone other than his own wife mean violence?"); perceptions (starting factors and aggravation factors of IPV) consisting of 18 items such as "talking about marital life problems to others will cause discomfort, disagreement, and dispute," or "tolerance of the husband's violent behavior because of their children"; the enablers (communication skills and environmental factors) were 15 items such as "lack of money and low income in the family will cause discomfort, disagreement, and dispute," or "knowledge of strengths and weaknesses in controlling or continuing disputes"; and the nurturer factors comprised six items, for example, "my family is really trying to help me when I have disputes with my husband," or "there are persons other than family and friends (for example, the family counselor) to whom I have access when disputing my husband or if necessary." A 5-point Likert scale, ranging from 1 to 5 (1=strongly agree, 2=agree, $3=$ =undecided, 4=disagree, and 5=strongly disagree), was used to measure the constructs. The complete version of the questionnaire is attached to the manuscript as a supplementary document (Supplement 1). The tool was pre-tested and modified to suit the local context. We sent a questionnaire to a panel of 15 academic members consisting of experts in the fields of psychology, health education, and violence against women to assess the validity of the content. Cronbach's $\alpha$ test was used to determine the reliability of the PEN-3 model constructs of the questionnaire in the original study $(r=0.82, n=456)$.

A screening instrument used to identify IPV among pregnant women was the CTS2 (the revised version of original Conflict Tactics Scales) developed by Straus. ${ }^{16)}$ This instrument was validated and translated into the local language prior to the commencement of the study. ${ }^{17}$ ) Data were collected using face-to-face interviews at baseline and 3 months after the intervention by midwives. Midwives and participants were blinded concerning the condition allocation.

\section{Intervention}

The experimental group received five sessions of public health educations from a clinical psychologist for 5 weeks at the health care clinic. The intervention program aimed at controlling and preventing IPV in pregnant women consisted of the following strategies: (1) Women were instructed to identify IPV using the trigger factors that aggravate it. The participants received a booklet containing information about IPV and conflict management techniques. A group discussion was held about the preventive measures against IPV. Women were encour- aged to share their experiences after watching a local movie about domestic violence. (2) The women received consultation to improve their communication by developing interpersonal skills. Counselors attempted to provide a safe learning environment to deliver educational courses to develop communication skills followed by an interactive discussion. (3) Contact details of the researchers were provided and the women were encouraged to contact and seek advice to manage their family issues as needed. (4) The women were also urged to actively seek a supportive environment among friends and family members, particularly with their mothers. They were also asked to develop their relationship with the support group at the clinic and the research team during the study period. Compassionate counseling, empathetic listening opportunities, and distribution of gift cards and books by the research team were among other supportive techniques utilized to encourage participation. We also attempted to facilitate consultation meetings with a private psychological counselor for individual face-toface sessions. Health care professionals working in the clinics were asked to be on the alert to accommodate consultation sessions for study participants when necessary. (5) At the end of each meeting, pregnant women were encouraged to allow a counselor to visit their house and provide a free consultation session with their husband.

Each stage of the intervention was carefully prepared to accommodate cultural norms and beliefs. All the educational materials, exercises, quotes, and information resources were selected carefully based on the community language.

One of the researchers always attended each session to monitor and ensure that the intervention content was being delivered consistently according to the guidelines. To motivate women to participate in education courses, the experimental group received two SMS reminders 24 hours prior to each session. To further encourage their attendance at meetings, they were given a gift (US\$ 3.00) and two handbooks on marital skills (entitled "My loving life with my spouse" and "Preventing intimate partner violence" both in the local language). The control group received the standard care from health care providers.

\section{Variables}

The PEN-3 model is based on a theoretical framework that places a positive spin on social issues within a cultural setting to guide health education and interventions. The model contains three elements: perception of IPV, enablers, and nurturers. Perception refers to women's acceptance of IPV as a norm in the community. Perceptions of the causes of IPV were in three categories: knowledge, triggering factors, and aggravating/increasing factors of IPV ${ }^{18)}$ Enablers include excellent communication skills, better economic status, and referral to legal support resources that facilitate or impede IPV based on their level of availability. Nurturers refer to the degree to which beliefs and actions are influenced by extended family, friends, and the community. ${ }^{14)}$

\section{Data Analysis}

Data analysis was performed using SAS software ver. 20.0 (SAS Institute Inc., Cary, NC, USA). The participants' characteristics were ana- 
lyzed using descriptive statistics, such as frequencies, percentages for categorical variables, and means \pm standard deviation for continuous variables. In addition, intervention effectiveness was assessed by comparing changes in outcome measures between baseline and post-intervention assessments in the experimental and control groups using a paired t-test.

\section{RESULTS}

We collected pre-test data before the intervention from 150 pregnant women. The response rate for the post-test questionnaire was $81.3 \%$. Twenty-eight pregnant women had medical limitations (abortion and stillbirth), migrated out of the province, or decided to drop the study due to stigma associated with IPV. Therefore we calculated pre-protocol which included all study participants based on participants' allocation when they were randomized, and used this to mitigate potential selection bias. The analysis showed that the two groups participating and excluded from the study did not significantly differ in experiences of IPV ( $\mathrm{P}=0.96)$. The mean age of the participants was $31.21 \pm 5.74$ years in the intervention group and $28.41 \pm 5.03$ years in the control group.

Of the participants in both groups, most women had secondary education and most husbands had less than 9 years of education. There were no statistically significant differences between the experimental and control group in terms of women and husbands' education, husband's employment status (stable/unstable job [A "stable job" is similar, with the added benefit that the work is usually continuous. There are no sudden layoffs or labor strife.]), monthly income (insufficient/ sufficient ["Insufficient income" means they live below the poverty line. The poverty threshold in Iran is defined as a daily income of less than US\$2.00 per person.]), ethnicity differences between couples, and health insurance status (Table 1). Furthermore, there were no statistically significant differences between the two groups at baseline.

Tables 2 and 3 show the mean and standard deviation of outcome variables (knowledge, perceptions [starting factors/aggravation factors], enablers [communication skills/environmental], and nurturers, as well as the prevalence of physical, psychological, and sexual vio- lence in the experimental and control groups). The intervention increased the mean score of the following constructs: knowledge $(\mathrm{P}<0.01)$, communication skills $(\mathrm{P}=0.04)$, nurturers $(\mathrm{P}=0.03)$, and sexual violence $(\mathrm{P}=0.04)$.

\section{DISCUSSION}

The present study was conducted to examine the impact of preventive interventions on IPV among pregnant women residing in slum areas. We found that intervention was effective in increasing the score of knowledge, enablers, and nurturers. The impact of the intervention was borderline in terms of the reduction of physical and psychological violence, but significant for the reduction in sexual violence.

Table 1. Characteristics of the study participants in the intervention and control groups

\begin{tabular}{lccc}
\hline \multicolumn{1}{c}{ Characteristic } & $\begin{array}{c}\text { Intervention } \\
\text { group }(\mathrm{n}=42)\end{array}$ & $\begin{array}{c}\text { Control group } \\
(\mathrm{n}=80)\end{array}$ & P-value \\
\hline $\begin{array}{ccc}\text { Woman's education (y) } \\
<9\end{array}$ & $16(38.1)$ & $33(41.3)$ & 0.93 \\
$9-12$ & $22(52.4)$ & $36(45.0)$ & \\
$>12$ & $4(9.5)$ & $11(13.8)$ & \\
Man's education (y) & & & 0.47 \\
$<9$ & $26(61.9)$ & $40(55.0)$ & \\
$9-12$ & $9(21.4)$ & $28(35.0)$ & \\
$>12$ & $7(16.7)$ & $12(15.0)$ & \\
Husband's employment status & & & 0.62 \\
$\quad$ Unstable job & $23(54.8)$ & $42(52.5)$ & \\
$\quad$ Stable job & $19(45.2)$ & $38(47.5)$ & \\
Monthly income & $12(28.5)$ & $24(30.0)$ & \\
Insufficient & $30(71.5)$ & $56(70.0)$ & \\
Sufficient & & & 0.77 \\
Ethnic differences couple & $10(23.8)$ & $17(21.2)$ & \\
$\quad$ Yes & $32(76.2)$ & $63(78.8)$ & \\
$\quad$ No & & & 0.42 \\
Health insurance status & $31(73.8)$ & $59(73.8)$ & \\
$\quad$ Yes & $11(26.2)$ & $21(26.2)$ & \\
No & & & \\
\hline
\end{tabular}

Values are presented as number (\%).

Table 2. Comparison of the mean and standard deviation of variables in the PEN-3 model and outcome of intimate partner violence before and after the intervention for both groups

\begin{tabular}{|c|c|c|c|c|c|c|}
\hline \multirow{2}{*}{ Variable } & \multicolumn{3}{|c|}{ Baseline } & \multicolumn{3}{|c|}{ After 3-month follow-up } \\
\hline & Intervention group & Control group & P-value & Intervention group & Control group & P-value \\
\hline Knowledge & $4.86 \pm 3.49$ & $4.82 \pm 3.09$ & 0.96 & $7.50 \pm 2.65$ & $5.14 \pm 3.51$ & 0.01 \\
\hline Starting factors & $29.93 \pm 9.54$ & $31.72 \pm 7.41$ & 0.23 & $33.19 \pm 6.14$ & $32.06 \pm 8.53$ & 0.45 \\
\hline Aggravation factors & $15.43 \pm 3.21$ & $15.25 \pm 3.38$ & 0.78 & $14.95 \pm 2.92$ & $15.01 \pm 3.22$ & 0.91 \\
\hline Enablers & $15.76 \pm 4.78$ & $15.46 \pm 4.07$ & 0.72 & $18.38 \pm 4.25$ & $16.2 \pm 3.83$ & 0.04 \\
\hline Environmental & $19.14 \pm 2.78$ & $19.60 \pm 3.16$ & 0.44 & $19.48 \pm 3.32$ & $19.89 \pm 3.15$ & 0.51 \\
\hline Nurturers & $13.7 \pm 4.60$ & $13.19 \pm 4.40$ & 0.89 & $15.79 \pm 4.45$ & $13.40 \pm 4.57$ & 0.03 \\
\hline Physical violence & $1.07 \pm 2.74$ & $1.21 \pm 2.41$ & 0.14 & $0.74 \pm 2.28$ & $1.20 \pm 2.60$ & 0.06 \\
\hline Psychological violence & $3.76 \pm 5.46$ & $4.55 \pm 5.50$ & 0.31 & $2.80 \pm 4.10$ & $4.52 \pm 5.43$ & 0.06 \\
\hline Sexual violence & $0.48 \pm 1.13$ & $0.54 \pm 1.19$ & 0.76 & $0.11 \pm 0.58$ & $0.61 \pm 1.22$ & 0.04 \\
\hline Financial violence & $0.29 \pm 0.85$ & $0.61 \pm 1.09$ & 0.06 & $0.24 \pm 0.62$ & $0.58 \pm 1.08$ & 0.15 \\
\hline
\end{tabular}

Values are presented as mean \pm standard deviation. 
Table 3. Comparison of the mean and standard deviation of variables in the kind of intimate partner violence before and after the intervention for both groups

\begin{tabular}{|c|c|c|c|c|c|c|c|c|}
\hline \multirow{2}{*}{ Types of violence } & \multicolumn{4}{|c|}{ Baseline } & \multicolumn{4}{|c|}{ After 3-month follow-up } \\
\hline & Intervention group & Control group & t-value & P-value & Intervention group & Control group & t-value & P-value \\
\hline Physical & $1.07 \pm 2.74$ & $1.21 \pm 2.41$ & -1.46 & 0.14 & $0.74 \pm 2.28$ & $1.20 \pm 2.60$ & -1.90 & 0.06 \\
\hline Psychological & $3.76 \pm 5.46$ & $4.55 \pm 5.50$ & -1.02 & 0.31 & $2.80 \pm 4.10$ & $4.52 \pm 5.43$ & -1.85 & 0.06 \\
\hline Sexual & $0.48 \pm 1.13$ & $0.54 \pm 1.19$ & -0.30 & 0.76 & $0.11 \pm 0.58$ & $0.61 \pm 1.22$ & -2.41 & 0.04 \\
\hline Financial & $0.29 \pm 0.85$ & $0.61 \pm 1.09$ & -1.91 & 0.06 & $0.24 \pm 0.62$ & $0.58 \pm 1.08$ & -1.45 & 0.15 \\
\hline
\end{tabular}

Values are presented as mean \pm standard deviation.

The interventions discussed in the literature vary in terms of accommodating the needs of victimized women and even their children. The literature focuses on one or more of the following interventions: providing shelter houses for the victim and their children, facilitating contact with authorities (e.g., the police), ${ }^{18)}$ presenting individual and group counseling, ${ }^{19)}$ and couple behavioral therapy. Most of these interventions are conducted in developed countries, while in low income or developing countries, the effectiveness of such interventions remains understudied. ${ }^{20)}$

In the present study, the intervention did not significantly reduce the physical, psychological, and financial violence against pregnant women. However, the first branch of the PEN-3 model, the knowledge of pregnant women, was increased. Increased awareness and education can act as a protective factor in preventing or reducing violence, perhaps in the long run. Low levels of education among study participants was a significant challenge in our study in enhancing the knowledge, perceptions, self-confidence, and communication skills of pregnant women. ${ }^{21)}$ The women's attitudes about the infliction of violence on them stem from traditional norms that guide them to accept the violence imposed on them by their husbands as a typical event in life. ${ }^{22)}$ These perceptions, in turn, lead to keeping the abuse a "family secret"; a secret that should not be revealed if one is a "good wife." In our study, participation in educational classes and interaction with others changed participants' behavior from passive to active. The expression of experiences from some women in a group discussion about fighting the violence led to other women disclosing this family secret giving them a voice to raise their concerns. ${ }^{21)}$

The enabling factors presented in the second domain of the PEN-3 model comprise two constructs called "communication skills" and "environment" and were entitled "enablers." Acquiring communication skills increased women's interactions with the outside world. Learning such skills supports effective coping to address the struggles and life situations rather than showing anger and resentment. It also increased participants' level of adaptation. ${ }^{21)}$ This attitude creates sympathy in the partner thus diminishes the violence. Studies have shown that supportive services offered in clinics and communities are far more effective than traditional and conventional counseling provided by members of the family (e.g., mothers or mothers-in-law). Moreover, women who receive supportive services are more likely to refer to police or local authorities for legal advice or the prosecution of perpetrators.
Nevertheless, the absence of government regulations or a specific plan which would facilitate inter-sectorial cooperation as well as a lack of legal permission for women to complain about their husbands, leads to an unsuccessful prosecution of abusers. ${ }^{23)}$ In Iran, in recent years, some indirect models of reporting have been developed, including premarital counseling, launching an investigation when family disputes are reported, a free 24-hour call service, establishing temporary shelters with on-call psychological counselors and finally, training women to improve their communication skills at high schools and universities. Nevertheless, women rarely report IPV to the legal system due to the lack of a supportive and robust authority to call on when the crisis occurs and the inefficiency of the judicial system. Even after women refer to the legal authorities, they are usually returned to their husbands and face even more stringent retaliation reactions and possibly harsher punishment by abusers. ${ }^{23)}$

Police officers, lawyers, judges, and prosecutors should be trained to prevent the severe consequences of IPV. Moreover, health care professionals treating and monitoring pregnant women should undergo extensive and universal training to plan effective interventions specific to each local population. ${ }^{24,25)}$

The third dimension of the PEN-3 model was the impact of the intervention on "nurturers." Nurturing the victims of IPV primarily requires the identification of these women. Cultural and social limitations in the region make difficult to identify such victims, hence obtaining intervention for such victims is nearly impossible. Nevertheless, health care providers, especially midwives and family health specialists, are trusted individuals and have an active role as nurturers in all health issues. Victims of IPV can and do occasionally reveal their secrets to health care professionals enabling them to reinforce healthy marital relationships, identify suspicious and risky relations, provide interventions for at-risk individuals, and follow-up vulnerable individuals or refer them to relevant authorities. Health care professionals are, therefore, very useful for nurturing victims of IPV. However, these professionals can neither act beyond the borders of their clinic nor in the households. Hence, when violence erupts in the house, women often find themselves alone and without any support. ${ }^{25)}$ Women who undergo IPV often report that members of their husband's family, or even their own family, act as allies to the perpetrators with the result that they believe they are the ones at fault and feel remorse for seeking help or speaking their mind against the violent husband. ${ }^{26)}$ This critical issue was seen as a social and cultural barrier preventing the coopera- 
tion and participation of perpetrators of domestic violence, namely husbands, to be an active participant in our research.

Our study revealed that the intervention was able to reduce sexual violence significantly. This might be due to the fact that the perpetrators consider reduction of desire during pregnancy and were more delicate in their behavior towards women. They stopped or reduced violence during pregnancy. The other important factor to consider is having more family support during prengnacy. ${ }^{24)}$

Sexual attitudes and behaviors in pregnancy are affected by a belief system influenced by ethnicity, culture, and religious ideology. Altering a person's mental image of their body, the sense of shame about sexual relations during pregnancy, and fear of damaging the fetus (e.g., the horror of abortion and premature delivery) can adversely influence the sexual response and eventually may lead to less sexual desire. ${ }^{25}$

Moreover, inadequate couples' knowledge about sexual relations during pregnancy, the existence of negative attitudes, and even superstitious beliefs about sexual issues during this period may lead to the reduction of sexual activities during pregnancy. This, in turn, may diminish affectionate feelings toward the spouse, causing anxiety and lack of self-confidence in the mother. ${ }^{26)}$ Our study attempted to educate women about these issues to mitigate such fears in women and guide them toward having a healthy and risk-free relationship. Future studies should focus on training the abusive partner about the potential causes of lack of sexual desire during pregnancy. ${ }^{27)}$

In conclusion, currently designed interventions based on the PEN-3 model had an impact on some but not all important variables because IPV is a complicated phenomenon unique to every culture and society. It is also crucial to prioritize slum areas for future suitable interventions to reduce IPV imposed during pregnancy. The results of this study could help decision-makers to identify the cultural appropriateness of interventions and develop effective policies to reduce IPV.

\section{CONFLICT OF INTEREST}

No potential conflict of interest relevant to this article was reported.

\section{FUNDING}

This study was supported by the Vice-Chancellor of Research and Technology and the Research Ethics Committee (Grant no., 1396.478).

\section{ACKNOWLEDGMENTS}

The authors would like to acknowledge the health clinic workers who allowed the study to be conducted on service centers. We would also extend our gratitude to the study participant, women living in slum areas around Hamedan.

\section{SUPPLEMENTARY MATERIALS}

Supplementary materials can be found via https://doi.org/10.4082/ kjfm.20.0118. Supplement 1. Questionnaire for assessing of the intimate partner violence based on PEN-3 model domains.

\section{ORCID}

Khadije Ezzati Rastegar: https://orcid.org/0000-0002-3503-5532

Babak Moeini: https://orcid.org/0000-0001-9376-0460

Forouzan Rezapur-Shahkolai: https://orcid.org/0000-0001-5049-1109

Asadollah Naghdi: https://orcid.org/0000-0002-6403-6948

Manoochehr Karami: https://orcid.org/0000-0002-0125-3523

Shayesteh Jahanfar: https://orcid.org/0000-0001-6149-1067

\section{REFERENCES}

1. Stockman JK, Hayashi H, Campbell JC. Intimate partner violence and its health impact on ethnic minority women [corrected]. J Womens Health (Larchmt) 2015;24:62-79.

2. O’Doherty L, Hegarty K, Ramsay J, Davidson LL, Feder G, Taft A. Screening women for intimate partner violence in healthcare settings. Cochrane Database Syst Rev 2015;2015:CD007007.

3. Bhandari S, Bullock LF, Bair-Merritt M, Rose L, Marcantonio K, Campbell JC, et al. Pregnant women experiencing IPV: impact of supportive and non-supportive relationships with their mothers and other supportive adults on perinatal depression: a mixed methods analysis. Issues Ment Health Nurs 2012;33:827-37.

4. Ahmadi R, Soleimani R, Jalali MM, Yousefnezhad A, Roshandel Rad M, Eskandari A. Association of intimate partner violence with sociodemographic factors in married women: a population-based study in Iran. Psychol Health Med 2017;22:834-44.

5. Tirado-Munoz J, Gilchrist G, Lligona E, Gilbert L, Torrens M. A group intervention to reduce intimate partner violence among female drug users: results from a randomized controlled pilot trial in a community substance-abuse center. Adicciones 2015;27:168-78.

6. Wood L. Hoping, empowering, strengthening: theories used in intimate partner violence advocacy. Affilia 2015;30:286-301.

7. Ayala Quintanilla BP, Taft A, McDonald S, Pollock W, Roque Henriquez JC. Social determinants and maternal exposure to intimate partner violence of obstetric patients with severe maternal morbidity in the intensive care unit: a systematic review protocol. BMJ Open 2016;6:e013270.

8. Gazmararian JA, Lazorick S, Spitz AM, Ballard TJ, Saltzman LE, Marks JS. Prevalence of violence against pregnant women. JAMA 1996;275: 1915-20.

9. Patro-Hernandez RM. Intimate partner violence against women: prevention and intervention strategies in Spain. In: Sanchez-Lopez MP, Liminana-Gras RM, editors. The psychology of gender and health: conceptual and applied global concerns. London: Academic Press; 2017. p. 199-229.

10. Soleimani A, Delpisheh A, Khademi N, Jafarinia B, Sayehmiri K. Prevalence of violence against women in during pregnancy in Iran: a sys- 
tematic review and meta-analysis. J Urmia Nurs Midwifery Fac 2016; 13:973-86.

11. Hoque ME, Hoque M, Kader SB. Prevalence and experience of domestic violence among rural pregnant women in KwaZulu-Natal, South Africa. South Afr J Epidemiol Infect 2009;24:34-7.

12. Chemuru NR, Srinivas SC. Application of the PEN-3 cultural model in assessing factors affecting adolescent pregnancies in Rural Eastern Cape, South Africa. Int J Reprod Fertil Sex Health 2015;S1:1-8.

13. Orrego Dunleavy V, Chudnovskaya E, Phillips J, McFarlane SJ. Applying the PEN-3 cultural model to address HIV/AIDS prevention in rural Guatemala. J Intercult Commun Res 2018;47:1-20.

14. Moeini B, Rezapur-Shahkolai F, Jahanfar S, Naghdi A, Karami M, Ezzati-Rastegar K. Utilizing the PEN-3 model to identify socio-cultural factors affecting intimate partner violence against pregnant women in Suburban Hamadan. Health Care Women Int 2019;40:1212-28.

15. Ekhtiari YS, Shojaeizadeh D, Foroushani AR, Ghofranipour F, Ahmadis B. Effect of an intervention on attitudes towards domestic violence among Iranian girls. J Pak Med Assoc 2014;64:987-92.

16. Straus MA. Cross-cultural reliability and validity of the Revised Conflict Tactics Scales: a study of university student dating couples in 17 nations. Cross-Cult Res 2004;38:407-32.

17. Adib-Hajbaghery M, Karimi R, Karbasi H, Haji-Rezaei M, Aminolroayaee E. Comparing violence against women with and without an addicted spouse in Kashan, Iran. Addict Health 2015;7:74-81.

18. Rivas C, Ramsay J, Sadowski L, Davidson LL, Dunne D, Eldridge S, et al. Advocacy interventions to reduce or eliminate violence and promote the physical and psychosocial well-being of women who experience intimate partner abuse. Cochrane Database Syst Rev 2015;(12): CD005043.
19. Jahanfar S, Howard LM, Medley N. Interventions for preventing or reducing domestic violence against pregnant women. Cochrane Database Syst Rev 2014;2014:CD009414.

20. Zlotnick C, Capezza NM, Parker D. An interpersonally based intervention for low-income pregnant women with intimate partner violence: a pilot study. Arch Womens Ment Health 2011;14:55-65.

21. Taghdisi MH, Estebsari F, Dastoorpour M, Jamshidi E, Jamalzadeh F, Latifi M. The impact of educational intervention based on empowerment model in preventing violence against women. Iran Red Crescent Med J 2014;16:e14432.

22. Lila M, Oliver A, Galiana L, Gracia E. Predicting success indicators of an intervention programme for convicted intimate-partner violence offenders: the Contexto Programme. Eur J Psychol Appl Leg Context 2013;5:73-95.

23. Bolhari J, Amiri T, Karimi Keisomi I, Mohseni Kabir M. Domestic violence prevention advocacy program: a pilot study in Tehran urban area. Iran J Psychiatry Clin Psychol 2018;24:124-35.

24. Rasoulian M, Jalali AH, Habib S, Nojomi MM, Jolfaei AG, Bolhari J. Risk factors of domestic violence in Iran. Iran J Psychiatry Behav Sci 2017;11:e4280.

25. Hugl-Wajek JA, Cairo D, Shah S, McCreary B. Detection of domestic violence by a domestic violence advocate in the ED. J Emerg Med 2012;43:860-5.

26. Coker AL, Bush HM, Cook-Craig PG, DeGue SA, Clear ER, Brancato $\mathrm{CJ}$, et al. RCT testing bystander effectiveness to reduce violence. Am J Prev Med 2017;52:566-78.

27. Fallah S, Rostamzade S, Ghelich M. Effective factors on confronting violence on married women. J Gorgan Univ Med Sci 2016;17:Pe91-6. 